UDC 338.512:51-77

LBC 65.054

\title{
DUAL TASK FOR THE ECOLOGICAL AND ECONOMIC INTER-INDUSTRY BALANCE MODEL WITH NON-LINEAR DEPENDENCE AND MONOTONOUSLY DECOMPOSABLE OPERATOR
}

\author{
Marina N. Pavlova \\ Polytechnic Institute (Branch) of Don State Technical University in Taganrog, Taganrog, Russian Federation \\ Tatyana A. Bednaya \\ Polytechnic Institute (Branch) of Don State Technical University in Taganrog, Taganrog, Russian Federation
}

Anna A. Borisova

Polytechnic Institute (Branch) of Don State Technical University in Taganrog, Taganrog, Russian Federation

\begin{abstract}
The paper deals with the dual task for the ecological and economic inter-industry balance model with non-linear dependencies which can be described by the system of non-linear equations with monotonously decomposable operators. The suggested model is the equilibrium prices model in which the costs of harmful wastage utilization and recycling are considered. Besides, there are non-linear interrelations between the branches of production, which allows predicting retail prices for the useful products. This is necessary for economistsanalysts who are engaged in forecasting prices of the manufactured products. The suggested algorithms are based on the application of the theory of duality and operational calculus. The authors study the conditions under which this model is solvable and has a nonnegative solution, if at the same time the given values can be negative. The methods of creation of bilateral estimated solutions are adapted for the model; the method of improvement of bilateral estimation is offered. Unlike the methods of searching the precise solution, the application of the method of bilateral estimation facilitates successful solution of tasks with big dimension of the processed models, without resorting to direct integration. The results of this paper can be used in solution of specific tasks of mathematics, economy, biology and other tasks with non-linear interrelations.
\end{abstract}

Key words: dual task, decomposable operators, wastes recycling, non-linear model, monotonous operators.

Citation. Pavlova M.N., Bednaya T.A., Borisova A.A. Dual Task for the Ecological and Economic Inter-Industry Balance Model with Non-Linear Dependence and Monotonously Decomposable Operator. Vestnik Volgogradskogo gosudarstvennogo universiteta. Seriya 3, Ekonomika. Ekologiya [Science Journal of Volgograd State University. Global Economic System], 2018, vol. 20, no. 3, pp. 42-49. (in Russian). DOI: https://doi.org/10.15688/jvolsu3.2018.3.5

\section{ДВОЙСТВЕННАЯ ЗАДАЧА К ЭКОЛОГО-ЭКОНОМИЧЕСКОЙ МЕЖОТРАСЛЕВОЙ БАЛАНСОВОЙ МОДЕЛИ С НЕЛИНЕЙНОЙ ЗАВИСИМОСТЬЮ И МОНОТОННО РАЗЛОЖИМЫМ ОПЕРАТОРОМ}

\author{
Марина Николаевна Павлова \\ Политехнический институт (филиал) ДГТУ в г. Таганроге, г. Таганрог, Российская Федерация
}


М.Н. Павлова, Т.А. Бедная, А.А. Борисова. Двойственная задача к эколого-экономической модели

Татьяна Алексеевна Бедная

Политехнический институт (филиал) ДГТУ в г. Таганроге, г. Таганрог, Российская Федерация

\section{Анна Анатольевна Борисова}

Политехнический институт (филиал) ДГТУ в г. Таганроге, г. Таганрог, Российская Федерация

Аннотация. В статье рассматривается двойственная задача к эколого-экономической модели с нелинейными зависимостями, которая описывается системой нелинейных уравнений с монотонно разложимыми операторами. Предложенная модель является моделью равновесных цен, в которой учитываются затраты на утилизацию ипереработку вредных отходов, причем между отраслями производства существуют нелинейные взаимосвязи, позволяющие прогнозировать розничные цены на полезные продукты, что необходимо для экономистов-аналитиков, занимающихся прогнозом цен на производимую продукцию. Предложенные алгоритмы базируются на применении теории двойственности и операционного исчисления. Авторами определены условия, при которых данная модель является разрешимой и имеет неотрицательное решение, если при этом заданные величины могут принимать отрицательные значения. Для модели адаптированы методы построения двусторонних оценок решения, предложен метод улучшения двусторонних оценок. В отличие от методов поиска точного решения применение метода двусторонних оценок способствует успешному решению задач с большой размерностью обрабатываемых моделей, не прибегая к непосредственному интегрированию. Результаты данной статьи могут быть использованы при решении конкретных задач математики, экономики, биологии и других задач с нелинейными взаимосвязями.

Ключевые слова: двойственная задача, разложимые операторы, переработка отходов, нелинейная модель, монотонные операторы.

Цитирование. Павлова М. Н., Бедная Т. А., Борисова А. А. Двойственная задача к эколого-экономической межотраслевой балансовой модели с нелинейной зависимостью и монотонно разложимым оператором // Вестник Волгоградского государственного университета. Серия 3, Экономика. Экология. - 2018. - Т. 20, № 3. - C. 42-49. - DOI: https://doi.org/10.15688/jvolsu3.2018.3.5

Известная в литературе [1] двойственная задача, построенная к линейной экономической модели, носит название модели равновесных цен. Эта модель позволяет прогнозировать цены на продукцию отраслей при известных значениях величин норм добавленной стоимости. Кроме того, с ее помощью прогнозируют изменение цен и инфляцию, являющиеся следствием изменения цены в одной из отраслей.

Больший интерес представляют модели с непрерывным временем. Построенная двойственная модель с непрерывным временем представляет собой систему нелинейных уравнений. Поскольку цены на продукцию не могут принимать отрицательные решения, то решения этих моделей должны быть неотрицательны. Поэтому нахождение условий, при которых модель будет разрешима и при этом иметь неотрицательное решение, является основной задачей. Затраты на выделение вредных отходов и их переработку влекут за собой появление в системе уравнений отрицательных величин. В результате исследуются системы, которые имеют неотрицательные решения при наличии отрицательных заданных параметров.
Ввиду большой отраслевой номенклатуры системы уравнений имеют очень большой порядок, что приводит к большой вычислительной сложности. Поэтому нахождение точного решения нелинейной модели межотраслевого баланса представляется крайне затруднительной задачей. Существенно облегчить эту задачу позволяет нахождение оценок неизвестного решения.

\section{1. Двойственная модель}

\section{для случая с непрерывным временем}

Модель с непрерывным временем, в которой учитывается выделение и переработка вредных отходов и внесение инвестиций на развитие производства:

$$
\begin{aligned}
& \left\{\begin{array}{l}
x(t)=A_{11} x(t)+\left(A_{12}^{(1)}-A_{12}^{(2)}\right) y(t)+ \\
+B_{11} \frac{d x(t)}{d t}+\left(B_{12}^{(1)}-B_{12}^{(2)}\right) \frac{d y(t)}{d t}+f_{1}(t), \\
y(t)=A_{21} x(t)+A_{12} y(t)+B_{21} \frac{d x(t)}{d t}+ \\
+B_{22} \frac{d y(t)}{d t}-f_{2}(t) .
\end{array}\right. \\
& x(t) \geq 0, y(t) \geq 0, \frac{d x(t)}{d t} \geq 0, \frac{d y(t)}{d t} \geq 0, \mathrm{t} \in[0 ; h] .
\end{aligned}
$$


Перейдем от модели с непрерывным временем к модели с дискретным временем. Как известно, при достаточно малых значениях $h$

$$
\frac{d x(t)}{d t} \approx \lim _{h \rightarrow 0} \frac{x_{t+h}-x_{t}}{h}, \frac{d y(t)}{d t} \approx \lim _{h \rightarrow 0} \frac{y_{t+h}-y_{t}}{h}
$$

и модель примет вид

$$
\left\{\begin{array}{l}
x_{t+h}=A_{11} x_{t+h}+\left(A_{12}^{(1)}-A_{12}^{(2)}\right) y_{t+h}+ \\
+B_{11} \frac{x_{t+h}-x_{t}}{h}+\left(B_{12}^{(1)}-B_{12}^{(2)}\right) \frac{y_{t+h}-y_{t}}{h}+f_{1, t+h}, \\
y_{t+h}=A_{21} x_{t+h}+A_{22} y_{t+h}+B_{21} \frac{x_{t+h}-x_{t}}{h}+ \\
+B_{22} \frac{y_{t+h}-y_{t}}{h}-f_{2, t+h} . \\
\quad x_{t+h} \geq 0, y_{t+h} \geq 0, x_{t} \geq 0, y_{t} \geq 0 .
\end{array}\right.
$$

Положим $h=1$, где под единицей подразумевается отчетный период,

$$
\begin{aligned}
A_{12}= & \left(A_{12}^{(1)}-A_{12}^{(2)}\right), B_{12}=\left(B_{12}^{(1)}-B_{12}^{(2)}\right), \\
g_{1}= & -B_{11} x_{t}-B_{12} y_{t}+f_{1, t+1}, g_{2}= \\
= & B_{21} x_{t}+B_{22} y_{t}+f_{2, t+1},
\end{aligned}
$$

тогда имеем

$$
\left\{\begin{array}{c}
x_{t+1}=\left(A_{11}+B_{11}\right) x_{t+1}+\left(A_{12}+B_{12}\right) y_{t+1}+g_{1}, \\
y_{t+1}=\left(A_{21}+B_{21}\right) x_{t+1}+\left(A_{22}+B_{22}\right) y_{t+1}-g_{2}, \\
x_{t+1} \geq 0, y_{t+1} \geq 0 .
\end{array}\right.
$$

Двойственная модель для (1.1), в которой учтены цены полезных продуктов и плата за выброс в окружающую среду вредных отходов, имеет вид

$$
\left\{\begin{array}{l}
p_{t+1}=\left(A_{11}+B_{11}\right) p_{t+1}+\left(A_{21}+B_{21}\right) q_{t+1}+u_{1}, \\
q_{t+1}=\left(A_{12}+B_{12}\right) p_{t+1}+\left(A_{22}+B_{22}\right) q_{t+1}-u_{2}, \\
p_{t+1} \geq 0, \quad q_{t+1} \geq 0,
\end{array}\right.
$$

если рассматривать частный случай модели двойственной к модели Леонтьева - Форда [4], когда $E_{x}=R^{n}, E_{y}=R^{m}$, и, следовательно,

$$
\begin{gathered}
E_{x}^{*}=R^{n}, \quad E_{y}^{*}=R^{m}, \quad p \in R^{n}, \\
q \in R^{m}, \quad u_{1} \in R^{n}, \quad u_{2} \in R^{m},
\end{gathered}
$$

где $E_{x}^{*}, \quad E_{y}^{*}$ - пространства линейных ограниченных функционалов, сопряженные к пространствам $E_{x}=R^{n}, E_{y}=R^{m}$, a $C_{i j}^{*}(i, j=1,2)$ сопряженные операторы к операторам $C_{i j}=\left(A_{i j}+B_{i j}\right)$.
Будем интерпретировать вектор $p$ как вектор цен полезных продуктов, то есть $i$-я $(1 \leq i \leq n)$ компонента вектора $p$ равна цене одной единицы $i$-го полезного продукта. Аналогично пусть $k$-я $(1 \leq k \leq m)$ компонента вектора $q$ равна плате за выброс в окружающую среду одной единицы $k$-го вредного продукта (загрязнения), являющегося побочным результатом производственной деятельности. Вектор $u_{1} \in R^{n}$ представляет собой вектор добавленных стоимостей, то есть компонента $u_{1}$ этого вектора указывает стоимость труда, затраченного на создание одной единицы $i$-го полезного продукта $(1 \leq i \leq n)$. Аналогично вектор $u_{2} \in R^{m}$ представляет собой вектор, $k$-я компонента которого равна уменьшению платы за загрязнение одной единицей $k$-го вредного продукта окружающей среды, в силу которой сама среда «борется» с загрязнением. Вектор $C_{11}^{*} p=p C_{11}$ - часть стоимости затрат по созданию единичного вектора полезного продукта, полученная за счет использования в производстве полезных продуктов всех отраслей в соответствующих количествах. Вектор $C_{21}^{*} q=q C_{21}$ - часть стоимости затрат при создании единичного вектора полезного продукта, связанная с мероприятиями по охране окружающей среды. Вектор $C_{12}^{*} p=p C_{12}=p\left(C_{12}^{(1)}-C_{12}^{(2)}\right)$ - часть затрат, направленных на борьбу с загрязнением окружающей среды $C_{12}^{(1)}$ и переработку отходов $C_{12}^{(2)}$, равная стоимости затраченных полезных продуктов при уничтожении и переработке единицы вредного продукта. Наконец, $C_{22}^{*} q=q C_{22}$ - часть затрат на борьбу с загрязнением, равная плате за побочный (вредный) результат этой деятельности, связанный с дополнительными выбросами в окружающую среду вредных продуктов при уничтожении единицы этого продукта (например, выброс в воздух вредных соединений при сжигании мусора), то есть создание новых групп вредных продуктов при уничтожении вредных отходов.

В последней модели, аналогично тому, как это было сделано ранее, переходим к непрерывному времени, считая, что единица это отчетный период.

Введем обозначения $u_{1}=s_{1}-B_{11} p_{t}-B_{21} q_{t}$, $u_{2}=s_{2}+B_{12} p_{t}-B_{22} q_{t}$, получим 
М.Н. Павлова, Т.А. Бедная, А.А. Борисова. Двойственная задача к эколого-экономической модели

$$
\left\{\begin{array}{l}
p_{t+h}=A_{11} p_{t+h}+A_{21} q_{t+h}+ \\
+B_{11} \frac{p_{t+h}-p_{t}}{h}+B_{21} \frac{q_{t+h}-q_{t}}{h}+s_{1, t+h}, \\
q_{t+h}=\left(A_{12}^{(1)}-A_{12}^{(2)}\right) p_{t+h}+A_{22} q_{t+h}+ \\
+\left(B_{12}^{(1)}-B_{12}^{(2)}\right) \frac{p_{t+h}-p_{t}}{h}+B_{22} \frac{q_{t+h}-q_{t}}{h}-s_{2, t+h}, \\
\quad p_{t+h} \geq 0, q_{t+h} \geq 0, p_{t} \geq 0, q_{t} \geq 0 .
\end{array}\right.
$$

Получили двойственную модель для модели (1.0).

$$
\left\{\begin{array}{l}
p(t)=A_{11} p(t)+A_{21} q(t)+ \\
+B_{11} \frac{d p(t)}{d t}+B_{21} \frac{d q(t)}{d t}+s_{1}, \\
q(t)=\left(A_{12}^{(1)}-A_{12}^{(2)}\right) p(t)+A_{22} q(t)+ \\
+\left(B_{12}^{(1)}-B_{12}^{(2)}\right) \frac{d p(t)}{d t}+B_{22} \frac{d q(t)}{d t}-s_{2}, \\
p(t) \geq 0, q(t) \geq 0 .
\end{array}\right.
$$

Преобразуем полученную модель. Обозначим:

$$
\begin{aligned}
& z=\left(\begin{array}{l}
p(t) \\
q(t)
\end{array}\right), \quad \frac{d z}{d t}=\left(\begin{array}{c}
\frac{d p(t)}{d t} \\
\frac{d q(t)}{d t}
\end{array}\right), \\
& f=\left(\begin{array}{r}
s_{1} \\
-s_{2}
\end{array}\right), A=\left(\begin{array}{ll}
A_{11} & A_{21} \\
A_{12}^{(1)} & A_{22}
\end{array}\right)-\left(\begin{array}{cc}
0 & 0 \\
A_{12}^{(2)} & 0
\end{array}\right) \text {, } \\
& B=\left(\begin{array}{ll}
B_{11} & B_{21} \\
B_{12}^{(1)} & B_{22}
\end{array}\right)-\left(\begin{array}{cc}
0 & 0 \\
B_{12}^{(2)} & 0
\end{array}\right), \\
& C=(E-A)^{-1} B, s=(E-A)^{-1} f \text {. }
\end{aligned}
$$

В этом случае двойственная модель перепишется следующим образом [3]:

$$
\begin{aligned}
& \left\{\begin{array}{l}
p(t)=\frac{d}{d t} C_{11} p(t)+\frac{d}{d t} C_{21} q(t)+s_{1}(t), \\
q(t)=\frac{d}{d t} C_{12}^{(1)} p(t)-\frac{d}{d t} C_{12}^{(2)} p(t)+\frac{d}{d t} C_{22} q(t)-s_{2}(t) .
\end{array}\right. \\
& C_{i j}(p)=\frac{d}{d t} C_{i j} p(t), C_{i j}(q)=\frac{d}{d t} C_{i j} q(t), i, j=1,2
\end{aligned}
$$

или при

$$
\left\{\begin{array}{l}
p=C_{11}(p)+C_{21}(q)+s_{1}, \\
q=C_{12}^{(1)}(p)-C_{12}^{(2)}(p)+C_{22}(q)-s_{2},
\end{array}\right.
$$

где $C_{i j}(p), C_{i j}(q) i, j=1,2$ - монотонные операторы.

\section{Экономический смысл}

Первое уравнение этой модели означает, что цена полезного продукта является справедливой, так как она состоит из стоимости затраченных полезных продуктов (как в процессе производства, так и в процессе борьбы с загрязнением окружающей среды) и добавленной стоимости, то есть стоимости затраченного труда. Второе соотношение модели (1.2) означает, что плата за создание одной единицы загрязнения есть сумма стоимости затраченного на борьбу с загрязнением полезного продукта и стоимости затрат на уничтожение воспроизводимого загрязнения и затрат на утилизацию. При этом назначенная плата за загрязнение является справедливой.

Необходимо выяснить условия, при которых система операторных уравнений (1.2) имеет неотрицательное решение $\left(p^{*}, q^{*}\right)$ при заданных неотрицательных векторах $s_{1} \geq 0, s_{2} \geq 0$. Если $\widetilde{E}$ - линейное полуупорядоченное пространство с сильно миниэдральным (существует точная верхняя (нижняя) грань ограниченного множества) нормальным (введена норма) конусом $\widetilde{K}\left[5\right.$, с. 52], операторы $C_{i j}(i, j=1,2)$ системы (1.2) вполне непрерывные монотонные и удовлетворяют условию Липшицุа

$$
\left\|C_{i j}(p)-C_{i j}(q)\right\| \leq k_{i j}\|p-q\|,(i, j=1,2),
$$

где $k_{i j}-$ константа, причем $k_{i j}$ таковы, что спектральный радиус $r(Q)<1$, где

$$
Q=\left(\begin{array}{ll}
k_{11} & k_{21} \\
k_{12} & k_{22}
\end{array}\right)
$$

Тогда система уравнений (1.2) имеет единственное решение $p=p^{*}, q=q^{*}$.

Действительно, пусть убывающие, ограниченные сверху последовательности $\bar{p}_{n}$ и $\bar{q}_{n}$, которые соответственно имеют пределы $\bar{p}$ и $\bar{q}$, и возрастающие, ограниченные снизу последовательности $\underline{p_{n}}$ и $\underline{q_{n}}$, пределы которых $\underline{p}$ и $q$ удовлетворяют системам

$$
\begin{aligned}
& \left\{\begin{array}{l}
\underline{p}=C_{11}(\underline{p})+C_{21}(\underline{q})+s_{1}, \\
\underline{q}=C_{12}^{(1)}(\underline{p})-C_{12}^{(2)}(\bar{p})+C_{22}(\underline{q})-s_{2} .
\end{array}\right. \\
& \left\{\begin{array}{l}
\bar{p}=C_{11}(\bar{p})+C_{21}(\bar{q})+s_{1}, \\
\bar{q}=C_{12}^{(1)}(\bar{p})-C_{12}^{(2)}(\underline{p})+C_{22}(\bar{q})-s_{2} .
\end{array}\right.
\end{aligned}
$$


Оценим норму разности первых уравнений систем (1.3)-(1.4)

$$
\begin{gathered}
\|\bar{p}-\underline{p}\|=\| C_{11}(\bar{p})+C_{21}(\bar{q})+s_{1}- \\
-C_{11}(\underline{p})-C_{21}(\underline{q})-s_{1} \| \leq \\
\leq\left\|C_{11}(\bar{p})-C_{11}(\underline{p})\right\|+\left\|C_{21}(\bar{q})-C_{21}(\underline{q})\right\| \leq
\end{gathered}
$$

используя условия Липшица

$$
\leq k_{11}\|\bar{p}-\underline{p}\|+k_{21}\|\bar{q}-\underline{q}\| .
$$

Сделаем оценку нормы разности вторых уравнений систем (1.3)-(1.4)

$$
\begin{gathered}
\|\bar{q}-\underline{q}\|=\left\|\begin{array}{l}
C_{12}^{(1)}(\bar{p})-C_{12}^{(2)}(\underline{p})+C_{22}(\bar{q})-s_{2}-\| \leq \\
-C_{12}^{(1)}(\underline{p})+C_{12}^{(2)}(\bar{p})-C_{22}(\underline{q})+s_{2}
\end{array}\right\| \\
\leq\left\|C_{12}^{(1)}(\bar{p})_{2}-C_{12}^{(1)}(\underline{p})\right\|+\left\|C_{12}^{(2)}(\bar{p})-C_{12}^{(2)}(\underline{p})\right\|+ \\
+\left\|C_{22}(\bar{q})-C_{22}(\underline{q})\right\| \leq\left(k_{12}^{(1)}-k_{12}^{(2)}\right)\|\bar{p}-\underline{p}\|+ \\
+k_{22}\|\bar{q}-\underline{q}\|=k_{12}\|\bar{p}-\underline{p}\|+k_{22}\|\bar{q}-\underline{q}\|,
\end{gathered}
$$

то есть

$$
\left\{\begin{array}{l}
\|\bar{p}-\underline{p}\|=k_{11}\|\bar{p}-\underline{p}\|+k_{21}\|\bar{q}-\underline{q}\|, \\
\|\bar{q}-\underline{q}\|=k_{12}\|\bar{p}-\underline{p}\|+k_{22}\|\bar{q}-\underline{q}\| .
\end{array}\right.
$$

Пусть $\bar{a}=(\|\bar{p}-\underline{p}\|,\|\bar{q}-\underline{q}\|)^{T}$, тогда система неравенств (1.5) запишется в виде

$$
\bar{a} \leq Q \bar{a}, \bar{a} \geq \Theta .
$$

Согласно [5, с. 73] из (1.6) вытекает

$$
\begin{gathered}
r(Q) \geq 1, \\
Q=\left(\begin{array}{cc}
k_{11} & k_{21} \\
k_{12}^{(1)}+k_{12}^{(2)} & k_{22}
\end{array}\right)=\left(\begin{array}{ll}
k_{11} & k_{21} \\
k_{12} & k_{22}
\end{array}\right) .
\end{gathered}
$$

Из неравенства (1.7) следует, что в случае, когда $\bar{a} \neq \Theta, r(Q) \geq 1$, где $r(Q)$ - спектральный радиус матрицы $Q$ (1.8). Это вытекает из теоремы об оценке спектрального радиуса положительного оператора по его поведению на фиксированном ненулевом элементе конуса $K$. Следовательно, если $r(Q)<1$, то $\bar{a}=\Theta$, а это значит $\bar{p}=p, \bar{q}=q$.

Убедимся в единственности решения.

Предположим, что существует несколько решений системы (1.2) $\left(p_{1} q_{1}\right)$ и $\left(p_{2}, q_{2}\right)$, удовлетворяющих системе (1.2):

$$
\begin{gathered}
\left\{\begin{array}{l}
p_{1}=C_{11}\left(p_{1}\right)+C_{21}\left(q_{1}\right)+s_{1}, \\
q_{1}=C_{12}^{(1)}\left(p_{1}\right)-C_{12}^{(2)}\left(p_{1}\right)+C_{22}\left(q_{1}\right)-s_{2} .
\end{array}\right. \\
\left\{\begin{array}{l}
p_{2}=C_{11}\left(p_{2}\right)+C_{21}\left(q_{2}\right)+s_{1}, \\
q_{2}=C_{12}^{(1)}\left(p_{2}\right)-C_{12}^{(2)}\left(p_{2}\right)+C_{22}\left(q_{2}\right)-s_{2} .
\end{array}\right.
\end{gathered}
$$

Из (1.9) путем вычитания (1.10), учитывая условия Липшица, получим следующее:

$$
\begin{gathered}
\left|p_{1}-p_{2}\right|=\mid C_{11}\left(p_{1}\right)+C_{21}\left(q_{1}\right)+s_{1}-C_{11}\left(p_{2}\right)- \\
-C_{21}\left(q_{2}\right)-s_{1}\left|\leq k_{11}\right| p_{1}-p_{2}\left|+k_{21}\right| q_{1}-q_{2} \mid
\end{gathered}
$$

Аналогично

$$
\begin{aligned}
\left|q_{1}-q_{2}\right| & \leq\left(k_{12}^{(1)}-k_{12}^{(2)}\right)\left|p_{1}-p_{2}\right|+k_{22}\left|q_{1}-q_{2}\right| \leq \\
& \leq k_{12}\left|p_{1}-p_{2}\right|+k_{22}\left|q_{1}-q_{2}\right| .
\end{aligned}
$$

В итоге

$$
\left\{\begin{array}{l}
\left|p_{1}-p_{2}\right| \leq k_{11}\left|p_{1}-p_{2}\right|+k_{21}\left|q_{1}-q_{2}\right|, \\
\left|q_{1}-q_{2}\right| \leq k_{12}\left|p_{1}-p_{2}\right|+k_{22}\left|q_{1}-q_{2}\right|
\end{array}\right.
$$

Если положить $\bar{a}=\left(\left|p_{1}-p_{2}\right|,\left|q_{1}-q_{2}\right|\right)^{T}$, то система неравенств (1.11) запишется в виде $\bar{a} Q \cdot \bar{a}, \bar{a} \geq \Theta$. Следовательно [5, с. 73],

$$
r(Q) \geq 1,
$$

где

$$
Q=\left(\begin{array}{cc}
k_{11} & k_{21} \\
k_{12}^{(1)}+k_{12}^{(2)} & k_{22}
\end{array}\right)=\left(\begin{array}{ll}
k_{11} & k_{21} \\
k_{12} & k_{22}
\end{array}\right) .
$$

Из неравенства (1.12) следует, что в случае, когда $\bar{a} \neq \Theta, r(Q) \geq 1$, где $r(Q)$ - спектральный радиус матрицы $Q$. Следовательно, если $r(Q)<1$, то $\bar{a}=\Theta$, а это значит, что $p_{1}=p_{2}, q_{1}=q_{2}$, то есть система (1.2) имеет единственное решение.

Таким образом, система уравнений (1.2) имеет на конусном отрезке решение $\left(p^{*}, q^{*}\right)$. Причем для этого решения выполняются неравенства

$$
u_{2} \leq \bar{p}, \underline{p} \leq u_{1}, v_{2} \leq \bar{q}, \underline{q} \leq v_{1} .
$$

Рассмотрим оценки решения. Если конус $\widetilde{K}$ правильный нормален [5, с. 36, 42], операто- 
ры $C_{i j}^{*}(i=1,2, j=1,2)$ вполне непрерывные и монотонные, а также существуют векторы $u_{i}, v_{i}$ такие, что $0 \leq u_{2} \leq u_{1}, 0 \leq v_{2} \leq v_{1}$ и выполняются условия

$$
\left\{\begin{array}{c}
u_{1} \geq C_{11}\left(u_{1}\right)+C_{21}\left(v_{1}\right)+s_{1}, \\
u_{2} \leq C_{11}\left(u_{2}\right)+C_{21}\left(v_{2}\right)+s_{1}, \\
v_{1} \geq C_{12}^{(1)}\left(u_{1}\right)-C_{12}^{(2)}\left(u_{2}\right)+C_{22}\left(v_{1}\right)-s_{2},(114) \\
v_{2} \leq C_{12}^{(1)}\left(u_{2}\right)-C_{12}^{(2)}\left(u_{1}\right)+C_{22}\left(v_{2}\right)-s_{2} .
\end{array}\right.
$$

Тогда система (1.2) имеет единственное решение $\left(p^{*}, q^{*}\right)$, причем

$$
u_{2} \leq p^{*} \leq u_{1}, v_{2} \leq q^{*} \leq v_{1}
$$

Системы (1.3)-(1.4) с учетом (1.13)(1.14), в силу непрерывности и монотонности операторов, запишутся:

$$
\begin{aligned}
& \left\{\begin{array}{l}
\underline{p}=C_{11}(\underline{p})+C_{21}(\underline{q})+s_{1} \leq C_{11}\left(u_{1}\right)+C_{21}\left(v_{1}\right)+ \\
+s_{1} \leq u_{1}, \\
\underline{q}=C_{12}^{(1)}(\underline{p})-C_{12}^{(2)}(\bar{p})+C_{22}(\underline{q})-s_{2} \leq C_{12}^{(1)}\left(u_{1}\right)- \\
-C_{12}^{(2)}\left(u_{2}\right)+C_{22}\left(v_{1}\right)-s_{2} \leq v_{1} .
\end{array}\right. \\
& \left\{\begin{array}{l}
\bar{p}=C_{11}(\bar{p})+C_{21}(\bar{q})+s_{1} \geq C_{11}\left(u_{2}\right)+C_{21}\left(v_{2}\right)+ \\
+s_{1} \geq u_{2}, \\
\bar{q}=C_{12}^{(1)}(\bar{p})-C_{12}^{(2)}(\underline{p})+C_{22}(\bar{q})-s_{2} \geq C_{12}^{(1)}\left(u_{2}\right)+ \\
+C_{12}^{(2)}\left(u_{1}\right)+C_{22}\left(v_{2}\right)-s_{2} \geq v_{2} .
\end{array}\right. \\
& u_{2} \leq \bar{p}, \underline{p} \leq u_{1}, v_{2} \leq \bar{q}, \underline{q} \leq v_{1} .
\end{aligned}
$$

Так как было установлено, что $\bar{p}=p$, $\bar{q}=\underline{q}$, то $u_{2} \leq p^{*} \leq u_{1}, v_{2} \leq q^{*} \leq v_{1}$, что совпадает с неравенствами (1.15).

\section{2. Двусторонние оценки решения двойственной балансовой модели}

В общей постановке модель (1.2) можно переписать в виде операторного уравнения с монотонно разложимым оператором $C(z)=$ $=C_{1}(z)-C_{2}(z)[2]$ :

$$
z=C_{1}(z)-C_{2}(z)+s
$$

Здесь $C_{1}(z), C_{2}(z)$ - монотонные операторы.

Операторное уравнение (2.1) целесообразно записать в виде

$$
z=\left(C_{1}-C_{2}\right)(z)+s
$$

где $\quad z=\left(\begin{array}{l}p \\ q\end{array}\right), \quad s=\left(\begin{array}{r}s_{1} \\ -s_{2}\end{array}\right), \quad C_{1}=\left(\begin{array}{ll}C_{11} & C_{21} \\ C_{12}^{(1)} & C_{22}\end{array}\right)$, $C_{1}=\left(\begin{array}{cc}0 & 0 \\ C_{12}^{(2)} & 0\end{array}\right)$.

По уравнению (2.2) построим новое уравнение

$$
\widetilde{z}=\widetilde{C}(\widetilde{z})+\widetilde{s},
$$

где $\widetilde{z}=\left(\begin{array}{l}x \\ y\end{array}\right), \widetilde{s}=\left(\begin{array}{l}S \\ S\end{array}\right), \widetilde{C}=\left(\begin{array}{cc}C_{1} & -C_{2} \\ -C_{2} & C_{1}\end{array}\right)$.

Элемент $\widetilde{z}$ можно рассматривать как элемент нового пространства $\widetilde{E}=(E, E)$ с нормой $\|\widetilde{z}\|=\|x\|+\|y\|$, полуупорядоченного конусом $K=(K,-K)$. При этом конус $\widetilde{K}$ «наследует» основные свойства конуса $K$ [2].

Оператор $\widetilde{C}$, очевидно, оставляет инвариантным конус $\widetilde{K}$, а это значит, что оператор $\widetilde{C}$ положителен относительно $\widetilde{K}$. С помощью изложенной схемы мы фактически можем сводить изучение уравнений с монотонно разложимыми операторами к изучению вспомогательного уравнения с оператором $\widetilde{C}$ в новом пространстве $\widetilde{E}$, при этом оператор $\widetilde{C}$ является положительным относительно.

Оператор $C_{1}$ является монотонным (то есть из $\left.x_{1} \leq x_{2} \Rightarrow C\left(x_{1}\right) \leq C\left(x_{2}\right)\right)$, а $C_{2}$ - антитонным (то есть из $x_{1} \leq x_{2} \Rightarrow C\left(x_{1}\right) \geq C\left(x_{2}\right)$ ) [2].

Пусть оператор $\widetilde{C}(\widetilde{z})$ уравнения (2.3) монотонен, непрерывен и вполне непрерывен, удовлетворяет условиям Липшица

$$
\left\|C_{i}(u)-C_{i}(v)\right\| \leq q_{L i}\|u-v\|,(i=1,2)
$$

и пусть $u_{k+1}=C_{1}\left(u_{k}\right)-C_{2}\left(v_{k}\right)+s, v_{k+1}=$ $=C_{1}\left(v_{k}\right)-C_{2}\left(u_{k}\right)+s$, причем $u_{0}, v_{0}$ удовлетворяют неравенствам $C_{1}\left(u_{0}\right)-C_{2}\left(v_{0}\right)+s \geq u_{0}$, $C_{1}\left(v_{0}\right)-C_{2}\left(u_{0}\right)+s \leq v_{0}$ и положим

$$
u_{k+1}^{*}=\frac{u_{k+1}+m_{k} v_{k+1}}{1+m_{k}}, v_{k+1}^{*}=\frac{v_{k+1}+m_{k} u_{k+1}}{1+m_{k}},
$$

где

$$
\begin{gathered}
m_{k}=\max \left\{m: u_{k+1}-u_{k} \geq m\left(v_{k}-v_{k+1}\right),\right. \\
\left.v_{k}-v_{k+1} \geq m\left(u_{k+1}-u_{k}\right)\right\} .
\end{gathered}
$$




\section{УПРАВЛЕНИЕ ЭКОНОМИЧЕСКИМ РАЗВИТИЕМ}

Тогда имеют место неравенства

$$
u_{0} \leq u_{1}^{*} \leq u_{k}^{*} \leq \ldots \leq z^{*} \leq \ldots \leq v_{k}^{*} \leq v_{1}^{*} \leq v_{0},
$$

и либо метод сходится за конечное число шагов, либо верна оценка:

$$
\left\|v_{k+1}^{*}-u_{k+1}^{*}\right\| \leq \frac{\chi-1}{\chi+1} q_{L}\left\|v_{k}-u_{k}\right\|
$$

$q_{L}-$ константа Липшица.

Действительно, покажем, что

$$
\begin{gathered}
u_{k+1}^{*} \leq C_{1}\left(u_{k+1}^{*}\right)-C_{2}\left(v_{k+1}^{*}\right)+s, \\
\text { a } v_{k+1}^{*} \leq C_{1}\left(v_{k+1}^{*}\right)-C_{2}\left(u_{k+1}^{*}\right)+s .
\end{gathered}
$$

На основании свойства линейности оператора имеем

$$
\begin{gathered}
C_{1}\left(u_{k+1}^{*}\right)-C_{2}\left(v_{k+1}^{*}\right)+s-u_{k+1}^{*}= \\
=C_{1}\left(\frac{u_{k+1}+m_{k} v_{k+1}}{1+m_{k}}\right)-C_{2}\left(\frac{v_{k+1}+m_{k} u_{k+1}}{1+m_{k}}\right)+s-u_{k+1}^{*}= \\
=\frac{1}{1+m_{k}}\left(C_{1}\left(u_{k+1}+m_{k} v_{k+1}\right)-C_{2}\left(v_{k+1}+m_{k} u_{k+1}\right)+\right. \\
\left.+s\left(1+m_{k}\right)-u_{k+1}-m_{k} v_{k+1}\right)= \\
=\frac{1}{1+m_{k}}\left(C_{1}\left(u_{k+1}\right)-C_{2}\left(v_{k+1}\right)+s+\right. \\
\left.+m_{k}\left(C_{1}\left(v_{k+1}\right)-C_{2}\left(u_{k+1}\right)+s\right)-u_{k+1}-m_{k} v_{k+1}\right)= \\
=\frac{1}{1+m_{k}}\left(\left(u_{k+2}-u_{k+1}\right)-m_{k}\left(v_{k+1}-v_{k+2}\right)\right) \geq \theta,
\end{gathered}
$$

так как $\left(u_{k+1}-u_{k}\right) \geq m\left(v_{k}-v_{k+1}\right)$, а следовательно,

$$
u_{k+1}^{*} \leq C_{1}\left(u_{k+1}^{*}\right)-C_{2}\left(v_{k+1}^{*}\right)+s .
$$

Аналогично можно показать, что

$$
v_{k+1}^{*} \leq C_{1}\left(v_{k+1}^{*}\right)-C_{2}\left(u_{k+1}^{*}\right)+s .
$$

Построение отрезка (2.4) можно рассматривать как один шаг рекуррентного процесса по уточнению двусторонних границ неизвестного решения $x^{*}$ исходного уравнения. Повторяя этот процесс достаточное число раз, мы в итоге получим метод построения двусторонних приближений, который можно рассматривать как метод ускорения сходимости двусторонних приближений в случае уравнения с монотонно разложимым оператором [2].
Рассмотрим норму разности $v_{k+1}^{*}$ и $u_{k+1}^{*}$

$$
\begin{aligned}
& \| \frac{1}{1+m_{k}}\left(C_{1}\left(v_{k}\right)-C_{2}\left(u_{k}\right)+s\right)+m_{k}\left(C_{1}\left(u_{k}\right)-C_{2}\left(v_{k}\right)+s\right)- \\
& -\frac{1}{1+m_{k}}\left(C_{1}\left(u_{k}\right)-C_{2}\left(v_{k}\right)+s\right)+m_{k}\left(C_{1}\left(v_{k}\right)-C_{2}\left(u_{k}\right)+s \mid \leq\right. \\
& \left.\leq\left|\frac{1-m_{k}}{1+m_{k}}\right||| C_{1}\left(v_{k}\right)-C_{1}\left(u_{k}\right)\|+\| C_{2}\left(v_{k}\right)-C_{2}\left(u_{k}\right) \|\right) \leq \\
& \leq\left|\frac{1-m_{k} \mid}{1+m_{k}}\right|\left(q_{1 L}\left\|v_{k}-u_{k}\right\|+q_{2 L}\left\|u_{k}-v_{k}\right\|\right),
\end{aligned}
$$

так как

$$
\begin{gathered}
\theta \leq\left\|C_{1}\left(v_{k}\right)-C_{1}\left(u_{k}\right)\right\|+\left\|C_{2}\left(v_{k}\right)-C_{2}\left(u_{k}\right)\right\| \leq \\
\leq\left(q_{1 L}+q_{2 L}\right)\left\|u_{k}-v_{k}\right\| .
\end{gathered}
$$

На основании [2] данное выражение $\left|\frac{1-m_{k}}{1+m_{k}}\right|$ меньше либо равно $\left|\frac{1-\chi}{1+\chi}\right|$.

Тогда

$$
\left\|v_{k+1}^{*}-u_{k+1}^{*}\right\| \leq\left|\frac{\chi-1}{\chi+1}\right| q_{L}\left\|v_{k}-u_{k}\right\|
$$

Таким образом, метод сходится со скоростью геометрической прогрессии [2].

\section{СПИСОК ЛИТЕРАТУРЫ}

1. Кремер, Н. Ш. Исследование операций в экономике: учеб. пособие для вузов / Б. А. Путко, И. М. Тришин, М. Н. Фридман. - М. : ЮНИТИ, 1997. $-407 \mathrm{c}$.

2. Кубекова, Б. С. Об одном методе построения двусторонних приближений к решению операторного уравнения с монотонно разложимым оператором / Б. С. Кубекова, М. Н. Павлова, В. Я. Стеценко // Журнал вычислительная математика и математическая физика. $-2001 .-$ Т. 41 , № 6. - C. 846-854.

3. Павлова, М. Н. Динамические балансовые модели с непрерывным временем с учетом экологического фактора и вложения инвестиций в развитие производства / М. Н. Павлова, Е. М. Петлина. Ростов н/Д : Издательский центр ДГТУ, 2012. - 100 с.

4. Павлова, М. Н. Модель отраслевого баланса, учитывающая экологический фактор / М. Н. Павлова. - Ставрополь : СКСИ, 2005. -60 с.

5. Стеценко, В. Я. Элементы теории полуупорядоченных пространств. Приближенное решение операторных уравнений / В. Я. Стеценко, И. А. Галкина. - Ставрополь : СГУ,1998. - 168 с. 


\section{REFERENCES}

1. Kremer N.H., Putko B.A., Trishin I.M., Fridman M.N. Issledovanie operatsiy v ekonomike [Operations Research in Economics]. Moscow, YuNITI Publ., 1997. $407 \mathrm{p}$.

2. Kubekova B.S., Pavlova M.N., Stetsenko V.Yu. Ob odnom metode postroeniya dvustoronnikh priblizheniy $\mathrm{k}$ resheniyu operatornogo uravneniya $\mathrm{s}$ monotonno razlozhimym operatorom [On a Method for Constructing Two-Sided Approximations to the Solution of an Operator Equation with a Monotonically Decomposable Operator]. Zhurnal vychislitelnaya matematika i matematicheskaya fizika, 2001, vol. 41, no. 6 , pp. 846-854.

3. Pavlova M.N, Petlina Ye.M. Dinamicheskie balansovyie modeli $s$ nepreryivnyim vremenem $s$ uchetom ekologicheskogo faktora i vlozheniya investitsiy v razvitie proizvodstva [Dynamic Balance Models with Continuous Time, Taking into Account the Environmental Factor and Investing in Production Development]. Rostov-on-Don, DGTU Publ., 2012. $100 \mathrm{p}$.

4. Pavlova M.N. Model otraslevogo balansa, uchityvayushchaya ekologicheskiy faktor [The Model of Industrial Balance Taking into Account the Ecological Factor]. Stavropol, SKSI Publ., 2005. $60 \mathrm{p}$.

5. StetsenkoV.Ya., Galkina I.A. Elementy teorii poluuporyadochennykh prostranstv. Priblizhennoe reshenie operatornykh uravneniy [Elements of the Theory of Partially Ordered Spaces. An Approximate Solution of Operator Equations]. Stavropol, SGU Publ., 1998. 168 p.

\section{Information about the Authors}

Marina N. Pavlova, Candidate of Sciences (Physics and Mathematics), Associate Professor, Polytechnic Institute (Branch) of Don State Technical University in Taganrog, Petrovskaya St., 109-a, 347904 Taganrog, Russian Federation, pavlova_mn@mail.ru.

Tatyana A. Bednaya, Candidate of Sciences (Engineering), Associate Professor, Polytechnic Institute (Branch) of Don State Technical University in Taganrog, Petrovskaya St., 109-a, 347904 Taganrog, Russian Federation, bednayat@mail.ru.

Anna A. Borisova, Candidate of Sciences (Pedagogy), Associate Professor, Polytechnic Institute (Branch) of Don State Technical University in Taganrog, Petrovskaya St., 109-a, 347904 Taganrog, Russian Federation, banet06021979@mail.ru.

\section{Информация об авторах}

Марина Николаевна Павлова, кандидат физико-математических наук, доцент, Политехнический институт (филиал) ДГТУ в г. Таганроге, ул. Петровская, 109-а, 347904 г. Таганрог, Российская Федерация, pavlova_mn@mail.ru.

Татьяна Алексеевна Бедная, кандидат технических наук, доцент, Политехнический институт (филиал) ДГТУ в г. Таганроге, ул. Петровская, 109-a, 347904 г. Таганрог, Российская Федерация, bednayat@mail.ru.

Анна Анатольевна Борисова, кандидат педагогических наук, доцент, Политехнический институт (филиал) ДГТУ в г. Таганроге, ул. Петровская, 109-а, 347904 г. Таганрог, Российская Федерация, banet06021979@mail.ru. 